\title{
BMJ Open Tuberculosis notification in a private tertiary care teaching hospital in South India: a mixed-methods study
}

\author{
Archana Siddaiah, ${ }^{1}$ Mohammad Naseer Ahmed, ${ }^{2}$ Ajay M V Kumar, ${ }^{3,4}$ \\ George D'Souza, ${ }^{5}$ Ewan Wilkinson, ${ }^{6}$ Thae Maung Maung, ${ }^{7}$ Rashmi Rodrigues ${ }^{1,8,9}$
}

To cite: Siddaiah A, Ahmed MN, Kumar AMV, et al. Tuberculosis notification in a private tertiary care teaching hospital in South India: a mixedmethods study. BMJ Open 2019;9:e023910. doi:10.1136/ bmjopen-2018-023910

- Prepublication history for this paper is available online. To view these files, please visit the journal online (http://dx.doi org/10.1136/bmjopen-2018023910).

Received 8 May 2018 Revised 31 October 2018 Accepted 5 November 2018

Check for updates

(c) Author(s) (or their employer(s)) 2019. Re-use permitted under CC BY. Published by BMJ.

For numbered affiliations see end of article.

Correspondence to Dr Archana Siddaiah; archanapink@yahoo.com

\section{ABSTRACT}

Objectives India contributes approximately $25 \%$ of the 'missing' cases of tuberculosis (TB) globally. Even though $\sim 50 \%$ of patients with TB are diagnosed and treated within India's private sector, few are notified to the public healthcare system. India's TB notification policy mandates that all patients with TB are notified through Nikshay (TB notification portal). We undertook this study in a private hospital to assess the proportion notified and factors affecting TB notifications. We explored barriers and probable solutions to TB notification qualitatively from health provider's perspective.

Study setting Private, tertiary care, teaching hospital in Bengaluru, South India.

Methodology This was a mixed-methods study. Quantitative component comprised a retrospective review of hospital records between 1 January 2015 and 31 December 2017 to determine TB notifications. The qualitative component comprised key informant interviews and focus groups to elicit the barriers and facilitators of TB notification.

Results Of 3820 patients diagnosed and treated, 885 (23.2\%) were notified. Notifications of sputum smearpositive patients were significantly more likely, while notifications of children were less likely. Qualitative analysis yielded themes reflecting the barriers to TB notification and their solutions. Themes related to barriers were: (1) basic diagnostic procedures and treatment promote notification; (2) misconceptions regarding notification and its process are common among healthcare providers; (3) despite a national notification system other factors have prevented notification of all patients; and (4) establishing hospital systems for notification will go a long way in improving notifications.

Conclusions The proportion of patients with TB notified by the hospital was low. A comprehensive approach both by the hospital management and the national TB programme is necessary for improving notification. This includes improving awareness among healthcare providers about the requirement for TB notifications, establishing a single notification portal in hospital, digitally linking hospital records to Nikshay and designating one person to be responsible for notification.

\section{BACKGROUND}

In 2016, approximately $40 \%$ of the estimated 10.4 million tuberculosis (TB) cases were

\section{Strengths and limitations of this study}

- A mixed-methods design where the qualitative component explains and complements the findings from the quantitative component.

- Retrospective nature of the quantitative component ensured that the study procedures did not influence the notifications.

- It is likely that both the proportions notified and the number of patients diagnosed or treated are marginal overestimates.

- The findings are limited by the quality of the records maintained.

'missing', that is, were undiagnosed or unreported. ${ }^{1}$ India contributes approximately $25 \%$ of the 'missing' cases globally. ${ }^{12}$ Finding these 'missing' cases and treating them successfully is vital to ending TB by 2030, as envisaged by the United Nations Sustainable Development Goals. ${ }^{34}$

Healthcare delivery in India involves both the public and private sectors. The Indian private healthcare sector is estimated to cater to approximately two-thirds of the inpatients and three-fourths of the outpatients in the country. ${ }^{5}$ The private healthcare sector also accounts for $54 \%$ of the healthcare teaching facilities in India. ${ }^{5}$ It is therefore not surprising that approximately two-thirds of the 2.2 million patients with TB annually are diagnosed and treated within the private healthcare sector. ${ }^{6}$ However, in 2017 only $19 \%$ of these patients receive care from, or are notified, that is, reported, to the Revised National Tuberculosis Control Programme (RNTCP), ${ }^{4} 7$ India's national health programme for the prevention and control of $\mathrm{TB}$, as compared with $81 \%$ from public sector. Though mandatory TB notification introduced in 2012 saw a sharp increase in TB notifications, notification from the private sector continues to be low. ${ }^{4-11}$ This is despite launching Nikshay, the case-based 
web-based national TB notification portal, accessible to all healthcare providers, laboratories and diagnostic facilities, both public and private, nationwide.

Improving the estimates of disease prevalence though is essential for planning, monitoring and evaluation of RNTCP. Yet barriers such as lack of time, poor awareness regarding notification, concern about breaching patient confidentiality, operational complexities in notifying, along with lack of trust in the public sector prevent complete notification. ${ }^{12-14}$

The information on the extent of notification from private tertiary care teaching facilities is limited. This study was designed to determine the proportion of $\mathrm{TB}$ cases notified and the factors that affect notification in a private tertiary care teaching healthcare facility in Karnataka State, South India. The study also explored qualitatively the gaps in the existing notification systems so as to enable the identification and development of strategies to improve notification.

\section{METHODS}

\section{Study design}

A mixed-methods study comprising a retrospective review of records to quantitatively assess the proportions of patients with TB notified, and a qualitative component to identify barriers to TB notification was used.

\section{Study setting}

The study was conducted at a private tertiary-level teaching hospital in Bengaluru, Karnataka State, South India. The hospital has 1250 beds and caters to approximately 2000 outpatients daily from diverse backgrounds. A network of laboratory, pathology and radiology services support the clinical departments at the hospital. TB-specific microbiological services available are microscopy, GenXpert MTB/RIF, solid culture, and liquid TB culture and drug susceptibility testing (such as mycobacterial growth indicator tube).

There is a computerised information system for these services and the pharmacy exists at the hospital. The Medical Records Department compiles and maintains inpatient and outpatient hospital records in paper format. Inpatient records are available electronically and outpatient records are available in paper format.

\section{The Indian RNTCP and its relationship with the study hospital}

The RNTCP, a vertical national health programme, strives to provide care and treatment at no cost to all patients with TB in India. The programme adheres to the diagnostic and treatment recommendations of the WHO. ${ }^{15}$ The programme delivers its services through a network of designated microscopy centre (DMC, population covered: 0.1 million) and peripheral health institutions (PHI) (primary, secondary and tertiary healthcare facilities including all healthcare academia). ${ }^{16}$

In addition, direct observed treatment (DOT) centres at PHIs are responsible for dispensing treatment, observing treatment doses swallowed (DOT), patient follow-up and patient retention in care. Until 2017, the RNTCP followed an alternate day treatment regimen, with DOT thrice a week in the intensive phase (2 months) and weekly once in the continuation phase (4 months). All public PHIs function as DOT centres and have a TB health visitor (TBHV), responsible for DOT and patient retention. DOT centres at academic institutions, however, have a medical officer in addition to the TBHV. A PHI may also function as DMC.

Even though the RNTCP sets guidelines it does not dictate diagnostic or treatment protocols to the private sector. However, it attempts to deliver public services to the private sector through public-private partnerships (PPP) and expects all private healthcare providers to notify patients with TB irrespective of a PPP through Nikshay.

\section{Management of TB at the hospital}

By virtue of being a private tertiary care teaching hospital the RNTCP has established a DMC and a DOT centre at the hospital through a PPP. The RNTCP staff at the study hospital therefore comprised aLaboratory technician (LT), a Medical Officer (MO) (position currently vacant) and a TBHV.

When diagnosed with TB at any of the clinical departments at the hospital, patients can choose to take antitubercular treatment (ATT) either through the DOT centre, at no cost, or through the hospital's pharmacy for a cost. The patient's physician guides the patient's choice of treatment on a case by case basis.

\section{Notification of patients with TB at the study hospital}

Irrespective of the source of treatment, all patients with TB who are diagnosed or treated at the hospital are expected to be referred to the DOT centres for registration with the RNTCP and subsequent notification via the online notification portal Nikshay. In the study hospital notification of patients with $\mathrm{TB}$ was the responsibility of the TBHV.

\section{Study population}

\section{Quantitative component}

Study subjects comprised all patients diagnosed with TB and/or treated for TB from 1 January 2015 to 31 December 2016. For this study, the definition of a patient with TB incorporated the RNTCP definitions and patients identified through pharmacy records. Pharmacy records served as a surrogate, especially for the outpatients diagnosed, in absence of outpatient electronic health records at the hospital. A patient with TB was therefore defined as (1) microbiologically confirmed (RNTCP): a patient with microbiologically confirmed TB using microscopy, bacterial culture and/or GenXpert MTB/RIF, or (2) clinically diagnosed (RNTCP): a patient with histopathological or radiological findings suggestive of $\mathrm{TB}$, irrespective of microbiological confirmation, or (3) a patient who availed 


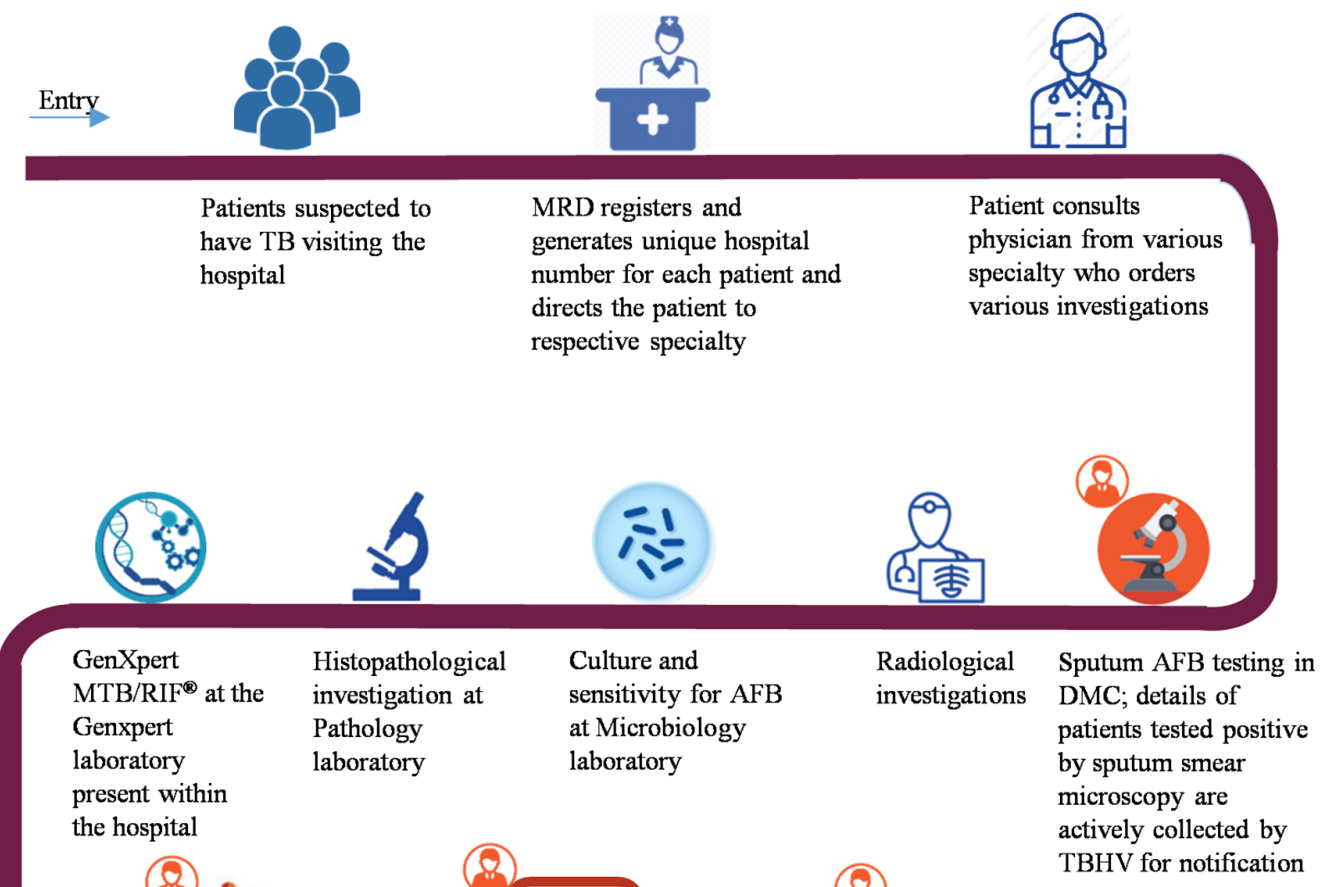

Blue-System set up within the hospital for TB diagnosis and treatment

Patients diagnosed with TB who are put on nonDOT purchase AKT4 from the hospital pharmacy

Patients get admitted in wards with their TB diagnosis coded as per ICD 10 and computerised and stored by the MRD; compiled by TBHV in the TB registers for notification

collect stations patient details collected by TBHV intermittently

Orange-System set up by the RNTCP programme within the hospital

(8) - Presence of TBHV (TB Health Visitor)

Figure 1 Flow of patients seeking TB care at a private tertiary care teaching hospital in Bengaluru, India. AFB, acid-fast bacilli; AKT4, anti-TB medication; DOT, direct observed treatment; ICD-10, International Classification of Diseases 10th Revision; MRD, Medical Records Department; RNTCP, Revised National Tuberculosis Control Programme; TB, tuberculosis; TBHV, TB health visitor.

ATT from the hospital's pharmacy identified through the pharmacy information system (PIS).

\section{Qualitative component}

Healthcare providers caring for patients with TB from various departments including clinicians, staff nurse, researchers, LT and TBHV were interviewed in-depth. Participants were chosen purposively to include those involved at various points within the $\mathrm{TB}$ case management cascade which is depicted in figure 1 .

\section{Data sources, variables and data collection procedures} Quantitative component

Demographic details of patients with TB such as patient's name, date of birth, gender, education, marital status, and residence (urban/rural), and year diagnosed, clinical department visited and source of the record were 
extracted from multiple sources. Data were first extracted from the inpatient electronic medical records database using the International Classification of Diseases 10th Revision (ICD-10) coding for TB (codes A15-A19). Subsequently data from the histopathology component of the laboratory information system (LIS) were extracted. For this, search terms such as 'tuberculosis', 'TB' and for possible typographical errors and 'lower and upper case formats' (eg, TB or tb) were used, as these diagnoses did not follow the ICD-10 coding. Data were similarly extracted from the radiology information system (RIS). These comprised reports from CT and MRI. Chest radiographs were not reported in the RIS as physicians review them in the light of clinical evidence for diagnosis. A laboratory or radiology report that read 'acid-fast bacilli (AFB) positive' or 'MTB detected' or 'strongly suggestive of TB' was considered as patients with TB. When in doubt, two physicians reviewed the reports and arrived at a consensus on the diagnosis. The PIS provided patient data for ATT purchased at the hospital's pharmacy.

Further, details of positive reports from sputum microscopy and culture registers were manually extracted and entered into Microsoft (MS) Excel as they were not available in the LIS.

A 'master database' for patients with TB diagnosed and/or treated in 2015 and 2016 was created using the unique hospital number (allocated to a patient at registration in the hospital) to match records and delete duplicate records in the various databases (PIS, LIS, RIS and manual registers).

A 'notification database' for patients with TB notified was also created. For this, data from the RNTCP register at the DOT centre of the hospital were entered into MS Excel. This was merged with data extracted from Nikshay portal. Patients diagnosed in late 2016 but who were notified in the first quarter of 2017 were also incorporated into this database.

In order to identify the proportions of TB cases notified, the 'master database' was matched with the 'notification database' using the VLOOKUP function in MS Excel. The patient's name was used as the primary matching variable. Records with a typographical mismatch in the patient's name were matched using a perfect match for 'sex' within an age range of \pm 3 years. Flow chart of data sources is depicted in figure 2.

\section{Qualitative component}

We conducted 11 in-depth interviews (IDI) with various healthcare providers and one focus group discussion (FGD) with 11 nursing staff. At the time of the study, nursing staff looked after activities such as reporting of diseases, and we conducted an FGD with them as they comprised a fairly homogeneous group of female healthcare providers and were therefore included in an FGD. The first author (AS), a physician trained in qualitative research, conducted the interviews. Two of the interviews

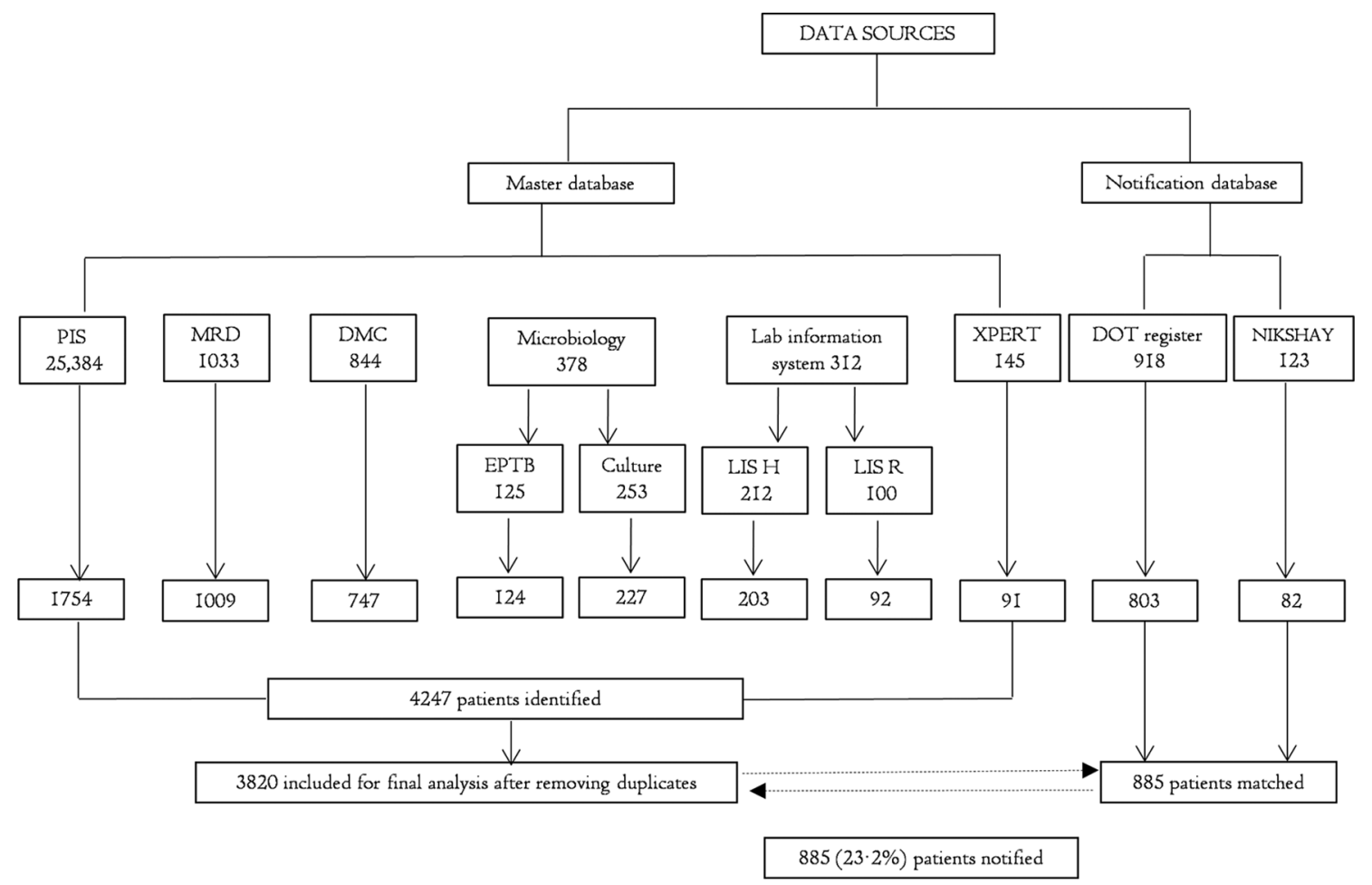

Figure 2 Flow chart showing various data sources and proportion of tuberculosis (TB) notified to Revised National Tuberculosis Control Programme (RNTCP) from a private tertiary care teaching hospital in Bengaluru, India, 2015-2016. DMC, designated microscopy centre; DOT, direct observed treatment; EPTB, extrapulmonary tuberculosis register; LIS H, laboratory information system-histopathology; LIS R, laboratory information system-radiology; MRD, Medical Records Department; PIS, pharmacy information system; Xpert, GenXpert MTB/RIF. 
were conducted in the local language, Kannada, and the rest in English. All interviews were audio recorded. A rapporteur made field notes during the interviews. After each interview, the key points were summarised and verified with the participants for validation. Data saturation guided the sample size. Each IDI lasted for 15-45 min and the FGD lasted for an hour.

\section{Data analysis}

\section{Quantitative component}

EpiData (V.2.2.2.186, EpiData Association, Odense, Denmark) and Stata (V.12.1, Texas, USA) software were used for data analysis. The proportion of patients with TB notified was the outcome variable. Associations (unadjusted) between the outcome variable and demographic and clinical characteristics were derived using the $X^{2}$ test. All bivariate associations with a 'p value' $<0.20$ were included in a log-binomial regression model to obtain adjusted prevalence ratios with $95 \%$ CIs. A 'p value' $<0.05$ was considered statistically significant.

\section{Qualitative component}

All interviews and field notes were transcribed and translated into English for analysis using the 'thematic framework approach'. The first and last author (AS, RR) familiarised themselves with a few transcripts and manually coded them. The codes were then compared and categorised based on similarity. This formed the framework for the analysis. ${ }^{17}$ The rest of the transcripts were subsequently indexed using the codes generated. Additional codes were added as and when necessary (box 1). The data were then summarised and mapped under various subthemes and themes which were reviewed by the rest of the authors for consensus.

\section{Patient and public involvement statement}

Patients were not involved in the design or conduct of the study.

\section{Ethics}

Ethics approval was obtained from the Institutional Ethics Committee, St John's Medical College, Bengaluru, Karnataka State, India, and the Ethics Advisory Group of the International Union Against Tuberculosis and Lung Disease, Paris, France. Permission to conduct the study and access hospital records was obtained from the chief of medical services at the hospital. Written informed consent was obtained from healthcare providers prior to interviews and included consent to audio record the interviews.

\section{RESULTS}

\section{Quantitative component}

A total of 3820 patients were diagnosed with TB and/ or treated during the study period. The demographic details of the patients with TB are described in table 1 . The median (IQR) age was $40(27-56)$ years and $7 \%$ of the patients were children $<15$ years of age.
Box 1 Thematic framework used for understanding the issues with TB notification at a private tertiary care teaching hospital, Bengaluru, India, 2015-2016

1.1. Gaps in the TB notification.1.1. Gaps in the TB notification. 1.2. Missing patients with TB.1.2. Missing patients with TB. 1.3. Confidentiality issue.1.3. Confidentiality issue. 2. Information to doctors.2. Information to doctors.

3. Disease disclosure to patients.3. Disease disclosure to patients.

4.1. TB diagnostic standard operating procedures.4.1. TB diagnostic standard operating procedures.

4.2. Technical issues associated with TB diagnosis.4.2. Technical issues associated with TB diagnosis.

5.1. Doctors' role in TB notification.5.1. Doctors' role in TB notification. 5.2. Reporting of patients with TB by doctors.5.2. Reporting of patients with TB by doctors.

5.3. Coordination between doctors and DOT centre.5.3. Coordination between doctors and DOT centre.

6.1. Standard operating procedures for TB notification.6.1. Standard operating procedures for TB notification.

6.2. Ease of notification.6.2. Ease of notification.

7.1. Policy decisions.7.1. Policy decisions.

8.1. Institute's notification policy.8.1. Institute's notification policy.

8.2. RNTCP notification policy.8.2. RNTCP notification policy.

8.3. Gaps in RNTCP notification policy.8.3. Gaps in RNTCP notification policy.

9.1. Streamlining TB notification.9.1. Streamlining TB notification.

9.2. Technological involvement.9.2. Technological involvement.

DOT, directly observed treatment short course; RNTCP, Revised National Tuberculosis Control Programme; TB, tuberculosis.

About a quarter of the patients received inpatient care and of them, nearly half were under the care of department of internal medicine, followed by chest medicine, neurology and paediatrics. About half of the patients with TB were identified through the pharmacy database while nearly $25 \%$ were identified through the LIS and laboratory registers.

Of the 3820 patients with TB, 885 (23.2\%, 95\% CI 21.9 to 24.5) were notified to the RNTCP. Of those notified, only $82(9 \%)$ were also recorded in the Nikshay portal. Factors associated with notification are shown in table 2. Notification was significantly lower (unadjusted analysis) in children, inpatients and patients identified through the LIS and PIS. Notification was significantly higher for patients whose diagnosis was confirmed microbiologically (sputum smear microscopy, culture or GenXpert MTB/ RIF). The final adjusted regression model showed age and sputum microscopy as determinants of notification.

\section{Qualitative component}

A total of 22 healthcare providers (11 from IDI and 11 from FGD) from various clinical departments at the hospital were interviewed. There were 10 physicians of whom seven were female. Six physicians had a work experience of $>10$ years. In addition, there were 12 paramedical staff including nurses, laboratory technicians and RNTCP staff most of whom had $>10$ years of work experience. 
Table 1 Demographic profile of patients with TB diagnosed and/or treated from 2015 to 2016 at a private tertiary care teaching hospital in Bengaluru, India

\begin{tabular}{lll}
\hline Variable & n (\%) & Notified (\%) \\
\hline Total & $3820(100)$ & $885(23.2)$
\end{tabular}

Age (years)

\begin{tabular}{llc}
$0-14$ & $264(6.9)$ & $24(9.1)$ \\
\hline $15-24$ & $476(12.5)$ & $118(24.8)$ \\
$25-34$ & $802(21.0)$ & $166(20.7)$ \\
$35-44$ & $670(17.5)$ & $159(23.7)$ \\
$45-54$ & $598(15.7)$ & $160(26.8)$ \\
$55-64$ & $503(13.2)$ & $129(25.6)$ \\
65 and above & $507(13.3)$ & $129(25.4)$
\end{tabular}

Sex

\begin{tabular}{|c|c|c|}
\hline Male & $2320(60.7)$ & $559(24.1)$ \\
\hline Female & 1500 (39.3) & $326(21.7)$ \\
\hline \multicolumn{3}{|l|}{ Residence } \\
\hline Within state & 2362 (61.8) & $567(24.0)$ \\
\hline Outside state & 1358 (35.5) & $293(21.6)$ \\
\hline Not available & $100(2.6)$ & $25(25.0)$ \\
\hline \multicolumn{3}{|l|}{ Marital status } \\
\hline Unmarried & 1008 (26.4) & $183(18.2)$ \\
\hline Married & 2604 (68.2) & $653(25.1)$ \\
\hline Others & $208(5.4)$ & $49(23.6)$ \\
\hline \multicolumn{3}{|l|}{ Year diagnosed } \\
\hline 2015 & 2071 (54.2) & $482(23.3)$ \\
\hline 2016 & $1749(45.8)$ & $403(23.0)$ \\
\hline \multicolumn{3}{|l|}{ Inpatient } \\
\hline Yes & 1009 (26.4) & $137(13.6)$ \\
\hline No & 2811 (73.6) & $748(26.6)$ \\
\hline \multicolumn{3}{|l|}{ Department $(n=1009)$} \\
\hline Medicine & $484(48.0)$ & $64(13.2)$ \\
\hline Pulmonary medicine & $141(14.0)$ & $21(14.9)$ \\
\hline Paediatrics & $81(8.0)$ & $16(19.8)$ \\
\hline Neurology & $88(8.7)$ & $5(5.7)$ \\
\hline General surgery & $41(4.1)$ & $5(12.2)$ \\
\hline Orthopaedics & $50(5.0)$ & $8(16.0)$ \\
\hline Others & 124 (12.3) & $15(12.1)$ \\
\hline \multicolumn{3}{|l|}{$\begin{array}{l}\text { Source of patients with } \\
\mathrm{TB}^{\star}\end{array}$} \\
\hline $\begin{array}{l}\text { Sputum microscopy } \\
\text { register }\end{array}$ & 747 (19.6) & $481(64.4)$ \\
\hline $\begin{array}{l}\text { Extrapulmonary TB } \\
\text { positive register }\end{array}$ & $124(3.2)$ & $24(19.4)$ \\
\hline $\begin{array}{l}\text { Histopathology } \\
\text { database }\end{array}$ & 203 (5.3) & $53(26.1)$ \\
\hline Radiology database & $92(2.5)$ & $13(13.7)$ \\
\hline Pharmacy database & 1754 (45.9) & $341(19.4)$ \\
\hline
\end{tabular}

Continued
Table 1 Continued

\begin{tabular}{lcc}
\hline Variable & n (\%) & Notified (\%) \\
\hline Culture register & $227(5.9)$ & $72(31.7)$ \\
$\begin{array}{l}\text { GenXpert MTB/RIF } \\
\text { register }\end{array}$ & $91(2.4)$ & $38(41.8)$ \\
Inpatient database & $1009(26.4)$ & $137(13.6)$ \\
\hline
\end{tabular}

${ }^{*}$ Cumulative percentage may add up to more than 100 since one patient could have tested positive by more than one diagnostic method.

TB, tuberculosis;

The four themes that emerged through the qualitative analysis were: (1) basic diagnostic modalities and treatment promote notification of TB; (2) misconceptions regarding notification and its process are common among healthcare providers; (3) despite a national notification system other factors prevented notification of all patients; and (4) establishing hospital systems for notification will go a long way in improving notifications (tables 3 and 4).

Basic diagnostic modalities and treatment promote notification of TB

Patients whose diagnosis was based on sputum microscopy and those receiving treatment through the RNTCP were more likely to be notified than those requiring complex diagnostics.

Patients who are sputum positive for TB bacteria are more likely to be notified

Diagnosis based on simple sputum smear microscopy was more likely to lead to notifications than patients requiring complex diagnostics such as radiography, biopsies, tissue examinations, bacteriological cultures or non-traditional laboratory diagnostics such as GenXpert MTB/ RIF and irrespective of whether these were inpatients or outpatients.

It was perceived that the RNTCP guidelines for notification restrict notification to only those patients diagnosed with MDR TB at an RNTCP accredited laboratory. Hence, patients with MDR TB were not notified.

Confirmation from [an Intermediate Reference Laboratory (IRL)] is a must for initiating the MDR regimen, without this MDR TB patients cannot be (treated with DOT) or notified. (Paramedical staff 9 (IDI) )

\section{Notifications are more likely for those diagnosed with pulmonary} $T B$

Most referrals to the RNTCP DOT centre were of patients diagnosed with pulmonary TB. Most patients with extrapulmonary TB were prescribed ATT through the hospital's pharmacy and therefore bypassed the DOT centre and hence notification. 
Table 2 Factors associated with TB notification at a private tertiary care teaching hospital in Bengaluru, India, from 2015 to 2016

\begin{tabular}{|c|c|c|c|c|c|c|}
\hline Variable & Total & $\begin{array}{l}\text { Notification } \\
\mathrm{n}(\%)\end{array}$ & $\begin{array}{l}\text { Crude PR } \\
(95 \% \mathrm{Cl}) \\
\end{array}$ & $P$ value & $\begin{array}{l}\text { Adjusted PR } \\
(95 \% \mathrm{Cl})\end{array}$ & $P$ value \\
\hline Total & 3820 & 885 (23.2) & - & - & - & - \\
\hline Age (years) & - & - & - & - & - & - \\
\hline Children $(<15)$ & 264 & $24(9.1)$ & 1 & - & 1 & - \\
\hline Adults $(\geq 15)$ & 3556 & 861 (24.2) & 2.6 (1.8 to 3.9$)$ & $0.000^{*}$ & 1.5 (1.0 to 2.2$)$ & $0.039^{\star}$ \\
\hline Sex & - & - & - & - & - & - \\
\hline Female & 1500 & $326(21.7)$ & 1 & - & - & - \\
\hline Male & 2320 & $559(24.1)$ & 1.1 (0.9 to 1.2$)$ & - & - & - \\
\hline Marital status & - & - & - & - & - & - \\
\hline Unmarried & 1008 & $183(18.2)$ & 1 & - & 1 & - \\
\hline Married & 2604 & $653(25.1)$ & 1.3 (1.1 to 1.5$)$ & $0.000^{\star}$ & 1.0 (0.9 to 1.2$)$ & 0.240 \\
\hline Others & 208 & 49 (23.6) & 1.2 (0.9 to 1.7$)$ & 0.066 & 1.1 (0.8 to 1.4$)$ & 0.346 \\
\hline Inpatient & - & - & - & - & - & - \\
\hline No & 2811 & 748 (26.6) & 1 & - & 1 & - \\
\hline Yes & 1009 & $137(13.6)$ & 0.4 (0.4 to 0.5$)$ & $0.000^{\star}$ & 1.0 (0.8 to 1.2$)$ & 0.925 \\
\hline Residence & - & - & - & - & - & - \\
\hline Within state & 2362 & $567(24.0)$ & 1 & & - & \\
\hline Outside state & 1358 & 293 (21.6) & 0.8 (0.7 to 1.0$)$ & 0.092 & - & - \\
\hline Not recorded & 100 & $25(25.0)$ & $1.0(0.7$ to 1.4$)$ & 0.819 & - & - \\
\hline Year diagnosed & - & - & - & - & - & - \\
\hline 2015 & 2071 & $482(23.3)$ & 1 & - & - & - \\
\hline 2016 & 1749 & $403(23.0)$ & 0.9 (0.8 to 1.1$)$ & 0.866 & - & - \\
\hline Sputum smear microscopy & - & - & - & - & - & - \\
\hline Positive & 747 & $481(64.4)$ & 4.8 (4.4 to 5.4$)$ & $0.000^{*}$ & 4.7 (4.1 to 5.3$)$ & $0.000^{*}$ \\
\hline Others & 3073 & $404(13.1)$ & 1 & - & 1 & - \\
\hline EPTB microscopy register & - & - & - & - & - & - \\
\hline Positive & 124 & $24(19.4)$ & 0.8 (0.5 to 1.1$)$ & 0.318 & - & - \\
\hline Others & 3696 & 861 (23.3) & 1 & - & - & - \\
\hline Culture & - & - & - & - & - & - \\
\hline Positive & 227 & $72(31.7)$ & 1.4 (1.1 to 1.7$)$ & $0.001^{*}$ & 1.0 (0.8 to 1.2$)$ & 0.855 \\
\hline Others & 3593 & $813(22.6)$ & 1 & & 1 & \\
\hline GenXpert MTB/RIF & - & - & - & - & - & - \\
\hline Positive & 91 & $38(41.8)$ & 1.8 (1.4 to 2.3 ) & $0.000^{\star}$ & 1.1 (0.9 to 1.3$)$ & 0.295 \\
\hline Others & 3729 & $847(22.7)$ & 1 & - & 1 & \\
\hline Histopathology database & - & - & - & - & - & - \\
\hline Present & 203 & $53(26.1)$ & $1.1(0.8$ to 1.4$)$ & 0.299 & - & - \\
\hline Others & 3617 & $832(23.0)$ & 1 & - & - & - \\
\hline Radiology database & - & - & - & - & - & - \\
\hline Present & 92 & $13(13.7)$ & $0.5(0.3$ to 0.9$)$ & $0.038^{*}$ & 0.7 (0.4 to 1.2$)$ & 0.285 \\
\hline Others & 3725 & $872(23.4)$ & 1 & - & 1 & - \\
\hline Pharmacy database & - & - & - & - & - & - \\
\hline Present & 1754 & $341(19.4)$ & 0.7 (0.6 to 0.8$)$ & $0.000^{*}$ & 0.9 (0.8 to 1.0$)$ & 0.839 \\
\hline Others & 2066 & $544(26.3)$ & 1 & & 1 & \\
\hline
\end{tabular}

${ }^{*}$ Significant $p$ value.

EPTB, extrapulmonary tuberculosis; PR, prevalence ratio; TB, tuberculosis. 
Table 3 Barriers and solutions identified for TB notification at a private tertiary care teaching hospital in Bengaluru, India, 2015-2016

\begin{tabular}{|c|c|}
\hline Barriers & Solutions \\
\hline $\begin{array}{l}\text { Patients with TB diagnosed } \\
\text { by culture, histopathology, } \\
\text { radiology, BAL usually } \\
\text { missed }\end{array}$ & Integration of LIS \\
\hline $\begin{array}{l}\text { Incomplete notification } \\
\text { among inpatients }\end{array}$ & $\begin{array}{l}\text { Triangulation of TB data from } \\
\text { all possible sources }\end{array}$ \\
\hline MDR TB missed & $\begin{array}{l}\text { Proper documentation and } \\
\text { communication which helps } \\
\text { in notification }\end{array}$ \\
\hline Lack of dedicated manpower & $\begin{array}{l}\text { Appointment of notification } \\
\text { officer }\end{array}$ \\
\hline Non-DOT not notified & $\begin{array}{l}\text { Referral of all patients started } \\
\text { on ATT by the treating doctor } \\
\text { to the notification officer }\end{array}$ \\
\hline Knowledge issues & $\begin{array}{l}\text { Awareness about notification } \\
\text { communicated }\end{array}$ \\
\hline Lack of capacity building & $\begin{array}{l}\text { Refresher trainings about } \\
\text { Nikshay }\end{array}$ \\
\hline $\begin{array}{l}\text { Absence of hospital } \\
\text { notification policy and } \\
\text { standard operating } \\
\text { procedure }\end{array}$ & Institutional notification policy \\
\hline $\begin{array}{l}\text { Inadequate networking } \\
\text { between stakeholders }\end{array}$ & $\begin{array}{l}\text { Having single notification } \\
\text { desk with dedicated } \\
\text { telephone number }\end{array}$ \\
\hline $\begin{array}{l}\text { Patient confidentiality } \\
\text { concerns }\end{array}$ & $\begin{array}{l}\text { Patient counselling about the } \\
\text { importance of notification, } \\
\text { ensuring adequate cyber } \\
\text { security }\end{array}$ \\
\hline Duplication of data & $\begin{array}{l}\text { Unique identifier (such as } \\
\text { social security number, } \\
\text { Aadhaar number in India) to } \\
\text { prevent duplication that helps } \\
\text { notify, track and retain patient } \\
\text { in care }\end{array}$ \\
\hline
\end{tabular}

ATT, anti-TB treatment; BAL, bronchoalveolar lavage; DOT, directly observed treatment short course; LIS, laboratory information system; MDR TB, multidrug-resistant tuberculosis.

Almost $85 \%$ extra-pulmonary patients don't take DOT or don't go to TBHV (who in turn notifies). (Paramedical staff 1 (IDI))

\section{Receiving treatment through the RNTCP is synonymous with notification}

Not all patients are initiated on DOT through the RNTCP. Some are prescribed ATT through the hospital's pharmacy at their own expense. As the responsibility for notification lies with the DOT centre, patients not referred to the DOT centre are not notified. Few medical and paramedical personnel knew the procedure for notification of $\mathrm{TB}$ at the hospital.
The physician that reviews the patients (reports do not advise DOT) so the RNTCP staff is not aware (of the patient diagnosed with TB). At least if the patients visit the DOT center, (the RNTCP staff) will know... but $50 \%$ of the patients treated by doctor are not referred to the DOT center. (Paramedical staff 1 (IDI)) Whoever goes to the DOT center (gets) registered and notified. (Physician 1 (IDI))

Misconceptions regarding notification and its process are common among healthcare providers

The level of knowledge and awareness regarding notification and its systems was poor. Healthcare providers did not perceive notification as their responsibility.

\section{Those who do not know, do not notify: awareness could improve notification}

Some healthcare providers were unaware that TB was a notifiable disease, others were unsure of the existing system for notifying TB and yet others presumed that notification was common knowledge. Out of 22 healthcare providers, 14 were aware of the RNTCP requirement of notification.

I don't think TB is a notifiable disease, is it a notifiable disease? That means every TB patient we come across (should be) notified? And to whom should we notify? (Physician 10 (IDI))

\section{Notification is someone else's responsibility}

There was confusion regarding the responsibility for notification. Many healthcare providers considered notification the responsibility of the RNTCP and not of the institution. The laboratories considered notification the responsibility of the treating physician and vice versa.

What we assume is that, the patient will go back to the doctor, maybe the doctor has to notify it. (Paramedical staff 5 (FGD))

I think from the labs they notify directly, we haven't taken it on us to notify as yet. (Physician 2 (IDI))

Despite a national notification system, other factors prevent notification of all patients

Inadequate training for using the notification portal Nikshay and mandatory information requirements within the portal were barriers to notification.

\section{Inadequate user training interferes with notifications via Nikshay portal}

There were mixed opinions regarding notification via Nikshay portal. While some considered Nikshay easy to use, few remembered having any training to use Nikshay for notification. Regular updates within the Nikshay portal without training to handle updates also interfered with notifications.

Nikshay is (quite) easy, what we had seen during the Nikshay demo, seemed okay. (Physician 2 (IDI)) 


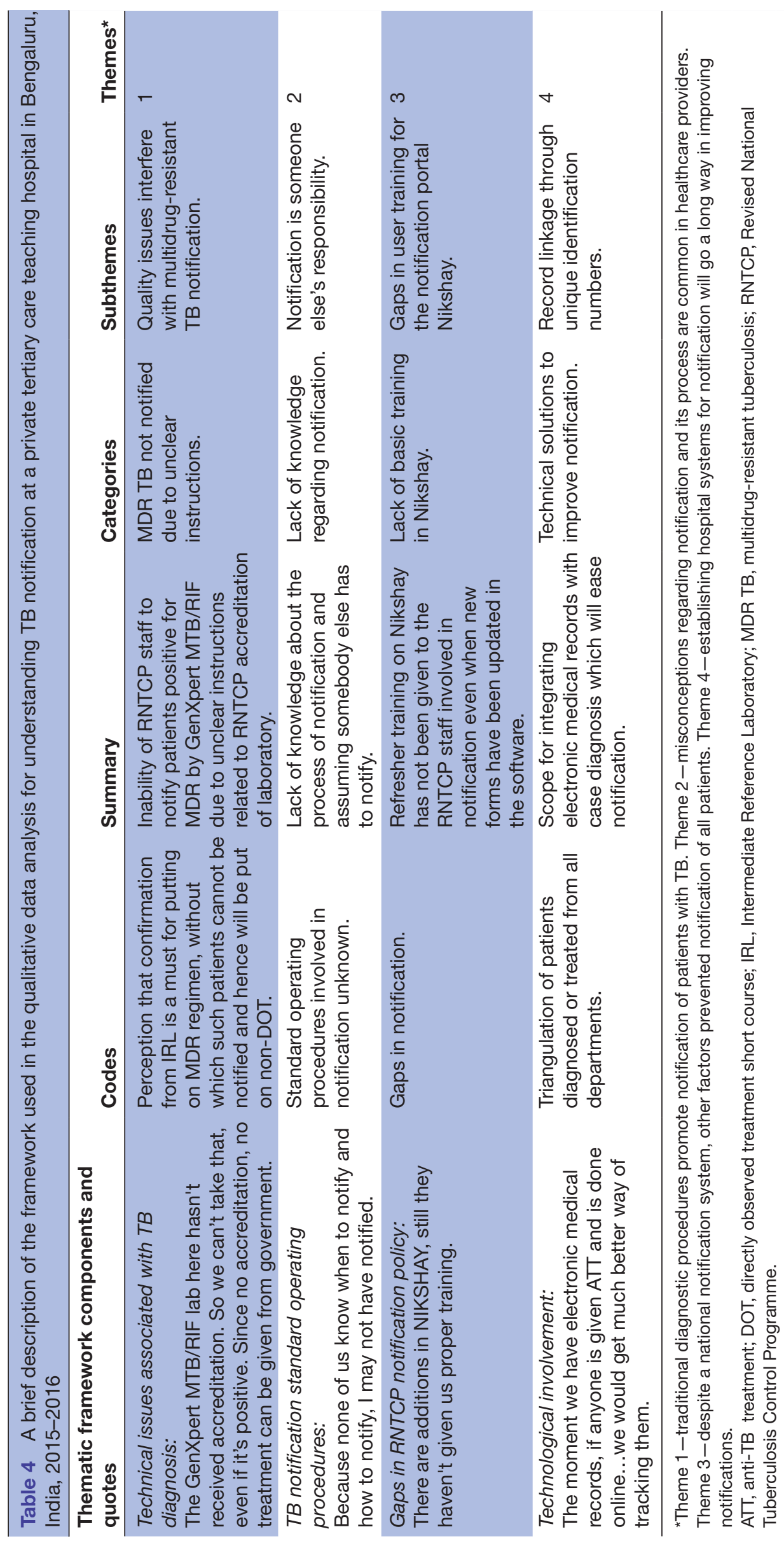


There are changes that are made to the Nikshay portal... they haven't trained us adequately for it. (Paramedical staff 10 (IDI))

\section{Fear of compromising privacy interferes with notification}

Fear of stigma from a breach in confidentiality prevents patients from sharing personal identifiers such as phone numbers. This limits entries into the notification portal due to missing information in 'mandatory fields'.

Establishing hospital systems for notification will go a long way in improving notifications

Notification policy, standard operating procedures and dedicated personnel supported with innovative technologies such as hot lines and mobile applications were suggested.

\section{Comprehensive institutional notification policy for TB: a necessity}

Developing and implementing a comprehensive institutional notification policy to improve notification was suggested. This policy was expected to provide guidance for delegating responsibilities and linking the various components of the hospital information system to enable identification and notification of patients with TB.

\section{Dedicated human resources could bridge gaps in the existing notification system}

Supplementing the existing human resource for notification, that is, TBHV and LT, with a dedicated notification officer (institutional) and an RNTCP medical officer at the DOT centre (via the programme) who could liaison with each other was considered essential.

Let's say, we appoint a person with an intercom or maybe a mobile (phone) so that the physician just calls that person and (informs)...then s/he could probably follow-up the patient to (obtain) the details.... (Physician 9 (IDI))

\section{Linking records through a unique identification number is useful} Documenting the hospital number in the RNTCP register and the government-issued unique identification number (Aadhaar number) ${ }^{18}$ in TB notification portal Nikshay could enable linkage while preventing duplication.

\section{Developing innovative information, communication and technology (ICT) support systems to aid notification}

A 'one window' concept, that is, establishing a dedicated notification hot line, or a mobile phone application to feed the details of patients with TB diagnosed and treated at the hospital, was suggested. Developing algorithms to short-list those diagnosed with TB from the LIS, along with electronic linking of outpatient, inpatient, laboratory, diagnostic and pharmacy records, was considered to support universal notification.

We have to go electronic and we have to then integrate everything...ordering (drug prescription) online...the moment we have electronic medical records... we would get much better way of tracking them. (Physician 7 (IDI))

\section{DISCUSSION}

Indian private healthcare sector contributes to only one-fifth of the TB notification in the country. ${ }^{9}$ Few reports have explored existing gaps in notification within the private sector. To our knowledge, this is the first report on the extent of TB notification and its challenges from a private tertiary care teaching hospital in India.

As in other studies, poor awareness and attitudes along with inadequate systems limited the TB notifications at the hospital to a quarter of those diagnosed ${ }^{19-21}$ Some private practitioners are of the opinion that notification of TB is unlikely to bring about change in prescription practices and question the need for collecting personal information that does not lead to public health action. ${ }^{14}$ Therefore, training and sensitisation of healthcare personnel for notification is recommended. Such training should focus on the benefits of notification from the public health and ethical perspective. ${ }^{13}$ It is also essential for the RNTCP to provide annual feedback to healthcare providers of the numbers notified and how this affects policy for TB care. Additionally, obtaining feedback from private practitioners regarding the notification process is expected to boost provider's morale and thereby notifications. ${ }^{11}$

Linking hospital records electronically could simplify notification. This does not eliminate manual data entry into the notification portal. Software solutions that feed data to the notification portal automatically could simplify notification and are currently being explored for MDR diagnostic machines. ${ }^{8}$ Further, applying ICD-10 codes for diagnoses, commonly used within TB notification systems globally, ${ }^{11}$ could standardise diagnoses, enable data capture through software systems and simplify notification.

The guidance for TB notification in India suggests the appointment of a TB nodal officer. ${ }^{8}$ The TBHV who currently fulfils this role in our context is probably overburdened with responsibilities in the absence of the 'DOT centre medical officer,' a functionary of the RNTCP. Reports from the private sector also indicate the need for additional human resources in the light of the volume of patients that they cater to. ${ }^{22}$ Identifying an additional 'nodal officer' for TB notification from among existing institutional personnel could optimise the use of existing resources for notification.

Healthcare providers suggested innovative ICTs such as mobile applications for notification. However, the short messaging service, interactive voice response or phone calls to notify TB enabled by the RNTCP for notification are not as popular as expected. Further, though, the Nikshay mobile application that is underway to simplify the notification process holds promise, ${ }^{23}$ whose effectiveness remains to be explored. 
Healthcare providers elsewhere in India recommend simplifying the existing notification technology to promote uptake. ${ }^{23}$ Regular training that includes Nikshay updates was widely requested, but currently negligible could remove existing technological barriers and enhance notification.

As the DOT centre at the hospital is located within the Chest Medicine Department, it is not surprising that sputum-positive patients are notified. Only $17 \%$ of chest physicians notified TB, reflecting the gap between awareness and action. ${ }^{12}$ However, in our study, ownership of the DOT centre probably made notification a responsibility of the chest physician and enhanced their engagement with the RNTCP. Locating DOT centres within clinical departments with the largest burden of patients with TB to improve notifications is worth exploring.

Though the Indian Academy of Paediatrics supports TB treatment through the RNTCP, ${ }^{24}$ the proportion of children with TB notified was low, reflecting the limited involvement of paediatricians in the RNTCP. The questionable bioavailability of paediatric ATT formulations and alternate day dosing schedules are known barriers to engaging paediatricians with the RNTCP. ${ }^{25}$ The introduction of the daily regimen with 'body weight bands' that inform dosing has the potential to improve provider engagement with the RNTCP and improve TB notifications thereof, irrespective of the patients' age. ${ }^{16}$ Also, creating a TB registry within each clinical department could improve departmentwise notifications.

Linking patient records using a unique identification number (Aadhaar number) ${ }^{18}$ or hospital number, and extending this system to involve the Nikshay portal could minimise duplication, simplify record and help retain patients in care. Studies indicate that patients are weary of sharing personal identifiers, that is, mobile phone numbers and address, for notification. ${ }^{10} 12$ This necessitates sensitising the general public of the need for mandatory disease notification through mass media campaigns and patients through counselling sessions. Further, perceived stigma prevents healthcare providers involved in notification uncomfortable with obtaining personal identifiers from patients. ${ }^{10}{ }^{12}$ Mobile phone numbers and the patient's address are mandatory fields in the Nikshay portal, without which notification is incomplete. Therefore, reminding healthcare providers of their obligation to obtain and report personal identifiers of patients with notifiable disease, as per the Indian Medical Council's Regulations 2002, ${ }^{26}$ might minimise discomfort in the light of responsibility. Simultaneously, mass media, posters and brochures placed in waiting rooms regarding notification could mitigate patients' fears with sharing personal identifiers.

Though punitive action for non-notification exists in India, it is not yet implemented. ${ }^{26}$ As in other TB high-burden countries, a recent mandate suggests that non-notification could result in heavy fines and even imprisonment. ${ }^{27}$ In the light of Government of India's politicoadministrative commitment towards TB control, punitive action is an eventuality that is best avoided. Therefore, at institutional level, enabling incentives for notification (tangible or intangible) and disincentives for non-notification ('warnings/memos', or monetary penalty) could reinforce the importance of notification. Further, the RNTCP provides a cash incentive of 250 INR to a 'private' healthcare provider for every patient with TB notified. ${ }^{28}$ Institutional proactiveness to ensure that its healthcare providers receive this incentive could also improve notifications.

Finally, testing for MDR TB cases in the study hospital was done using GenXpert MTB/RIF equipment that was acquired through the Initiative for Promoting Affordable and Quality TB Tests (IPAQT) project. IPAQT aims to bring WHO-approved TB tests at affordable prices to patients in private sector. ${ }^{29}{ }^{30}$ This was the only MDR TB diagnostic service available at the hospital. MDR TB cases detected through IPAQT are entered into Nikshay through a subportal within the hospital's primary Nikshay portal. It is only through this subportal that a person diagnosed with MDR TB at the hospital could be notified. A lack of awareness of this separate portal prevented notification of MDR TB diagnosed in the hospital. Understanding these issues at project initiation, documenting project procedures and ensuring 'complete knowledge transfer' to institutional personnel when institutions absorb such projects is necessary.

\section{Methodological issues}

The mixed-methods design with the quantitative and qualitative components validated and complemented each other. It is possible that our definition of notification overestimated the numbers notified. We were also liberal with our criteria for matching databases. However, we included all patients both diagnosed and treated at the hospital even if they availed a 'one-time' consultation. This probably also inflated the denominator minimising any overestimate.

The retrospective nature of the quantitative component meant that the study procedures did not influence changes in notification, as might have been observed if the study were prospective. Further, the quantitative component, based on a review of records, is limited by the quality of the data in the records, for example, we could not assess the association of the treating clinical department and treatment regimens on notification.

The study included healthcare providers who encountered patients with TB at different points in the hospital as represented in figure 1, including hospital staff and RNTCP staff. Therefore, we believe that this sample is fairly representative of those healthcare providers who manage patients with TB. This, along with a description of the study context and methodology, enables the reader to judge its applicability of the results to their context. The first author's position as a physician in the study setting helped her contextualise the results. Sharing the results with all authors with diverse backgrounds and skills improved the interpretation of the results, further improving generalisability. 


\section{CONCLUSIONS}

The low proportions of TB notifications at the hospital call for urgent action to identify strategies that can improve notification. A combined approach from within (managerial) and outside the institution (RNTCP) is necessary. Generating awareness regarding notification and developing a comprehensive notification policy along with establishing a notification portal is essential. Supplementing this with technological innovations such as mobile applications and expanding the scope of the existing hospital information system to capture outpatient data and link patient records is essential.

We also call on tertiary-level teaching hospitals both within India and globally to evaluate the TB notifications and its barriers in their setting. Such information is hoped to support the development of evidence-based strategies that enhance public-private engagement for TB notification and control.

\section{Author affiliations}

${ }^{1}$ Department of Community Health, St John's National Academy of Health Sciences, Bengaluru, Karnataka, India

${ }^{2}$ St John's Research Institute, St John's National Academy of Health Sciences, Bengaluru, Karnataka, India

${ }^{3}$ Operational Research, International Union Against Tuberculosis and Lung Disease, Paris, France

${ }^{4}$ Operational Research, International Union Against Tuberculosis and Lung Disease, New Delhi, India

${ }^{5}$ Department of Pulmonary Medicine, St John's National Academy of Health

Sciences, Bengaluru, Karnataka, India

${ }^{6}$ Institute of Medicine, University of Chester, Chester, UK

${ }^{7}$ Department of Medical Research, Ministry of Health and Sports, Yangon, Myanmar ${ }^{8}$ Department of Public Health Sciences, Karolinska Institute, Stockholm, Sweden

${ }^{9}$ Wellcome Trust/DBT India Alliance, Banjara Hills, Hyderabad, India

Acknowledgements This research was conducted through the Structured Operational Research and Training Initiative (SORT IT), a global partnership led by the Special Program for Research and Training in Tropical Diseases at the World Health Organization (WHO/TDR). The model is based on a course developed jointly by the International Union Against Tuberculosis and Lung Disease (The Union) and Medécins sans Frontières (MSF/Doctors Without Borders). The specific SORT IT programme which resulted in this publication was jointly developed and implemented by: The Union South-East Asia Office, New Delhi, India; the Center for Operational Research, The Union, Paris, France; The Union, Mandalay, Myanmar; MSF Luxembourg Operational Research (LuxOR); MSF Operational Center Brussels (MSF OCB); Institute of Medicine, University of Chester, UK; and Department of Medical Research, Ministry of Health and Sports, The Republic of The Union of Myanmar.

Contributors AS, GDS and RR conceived the study. AMVK and EW along with AS, GDS and RR designed the protocol. MNA did the literature search. AS and MNA did data entry and extracted the electronic data. AS undertook the interviews. AS, AMVK, RR and TMM analysed and interpreted the data. AS drafted the manuscript with support from AMVK and RR. GDS and EW critically revised the successive drafts. All authors have seen and approved the final version of this manuscript for publication.

Funding The training programme and open access publications costs were funded by the Department for International Development (DFID), UK and La FondationVeuve Emile Metz-Tesch (Luxembourg). The study is supported by grants from the Swedish Research Council and the Wellcome Trust/DBT India Alliance.

Disclaimer The funders had no role in study design, data collection and analysis, decision to publish, or preparation of the manuscript. The corresponding author (AS) confirms that she had full access to all the data in the study and had final responsibility for the decision to submit for publication.

Competing interests AS, GDS and RR are employed at the tertiary-level teaching facility that was evaluated in this study.
Patient consent Not required.

Ethics approval Institutional Ethics Committee, St John's Medical College and Hospital, and Ethics Advisory Group of the International Union Against Tuberculosis and Lung Disease, Paris, France.

Provenance and peer review Not commissioned; externally peer reviewed.

Data sharing statement Data will be made available with reasonable request from the corresponding author.

Open access This is an open access article distributed in accordance with the Creative Commons Attribution 4.0 Unported (CC BY 4.0) license, which permits others to copy, redistribute, remix, transform and build upon this work for any purpose, provided the original work is properly cited, a link to the licence is given, and indication of whether changes were made. See: https://creativecommons.org/ licenses/by/4.0/.

\section{REFERENCES}

1. World Health Organization. Global tuberculosis report. 2017 http:// apps.who.int/iris/bitstream/10665/259366/1/9789241565516-eng. pdf?ua=1 (Accessed 11 Dec 2017).

2. Subbaraman R, Nathavitharana RR, Satyanarayana S, et al. The tuberculosis cascade of care in india's public sector: a systematic review and meta-analysis. PLoS Med 2016;13:e1002149.

3. United Nations. Sustainable development goals: 17 goals to transform our world. 2015 http://www.un.org/sustainabledevel opment/health/ (Accessed 11 Dec 2017).

4. World Health Organization. The End TB Strategy. 2014 http://www. who.int/tb/End_TB_brochure.pdf (Accessed 11 Dec 2017).

5. Kumar S. Institute for studies in industrial development institute for studies in industrial development private sector in healthcare delivery market in INDIA: structure, growth and implications. 2015 http://isid. org.in (Accessed 27 Apr 2018).

6. Satyanarayana S, Nair SA, Chadha SS, et al. From where are tuberculosis patients accessing treatment in India? Results from a cross-sectional community based survey of 30 districts. PLoS One 2011;6:e24160.

7. Arinaminpathy $\mathrm{N}$, Batra $\mathrm{D}$, Khaparde $\mathrm{S}$, et al. The number of privately treated tuberculosis cases in India: an estimation from drug sales data. Lancet Infect Dis 2016;16:1255-60.

8. Central TB Division,Directorate General of Health Services,Ministry of Health \& Family Welfare G of I. Guidance for TB Notification in India. 2012 https://tbcindia.gov.in/showfile.php?lid=3139 (Accessed 11 Dec 2017).

9. Central TB Division, Directorate General of Health Services. TB India 2017. Revised national tuberculosis control programme annual status Report. 2017 https://tbcindia.gov.in/WriteReadData/TB India 2017. pdf (Accessed 11 Dec 2017).

10. Philip S, Isaakidis $P$, Sagili KD, et al. "They know, they agree, but they don't do"-the paradox of tuberculosis case notification by private practitioners in Alappuzha district, Kerala, India. PLoS One 2015;10:e0123286.

11. Uplekar M, Atre S, Wells WA, et al. Mandatory tuberculosis case notification in high tuberculosis-incidence countries: policy and practice. Eur Respir J 2016;48:1571-81.

12. Thomas BE, Velayutham B, Thiruvengadam K, et al. Perceptions of private medical practitioners on tuberculosis notification: a study from Chennai, South India. PLoS One 2016;11:e0147579.

13. Yeole RD, Khillare K, Chadha VK, et al. Tuberculosis case notification by private practitioners in Pune, India: how well are we doing? Public Health Action 2015;5:173-9.

14. Gawde N. Do we need notification of tuberculosis? A public health perspective. Indian J Med Ethics 2013;10:56-8.

15. World Health Organisation. Definitions and reporting framework for tuberculosis - 2013 revision. 2014.

16. Central TB Division G of I. Technical and operational guidelines for TB control in India: treatment of TB. https://tbcindia.gov.in/showfile. php?lid=3219 (Accessed 28 Apr 2018).

17. Ritchie J, Lewis J, McNaughton Nicholls C, et al. Qualitative research practice : a guide for social science students and researchers Sage. 2014 https://books.google.co.in/books?id=EQSIAwAAQBAJ\& printsec=frontcover\&source $=g b s \_g e \_s u m m a r y \_r \& c a d=0 \# v=$ onepage\&q\&f=false (Accessed 24 Aug 2017).

18. Ministry of Law and Justice G of I. The Aadhar (Targeted delivery of financial and other subsidies, benefits and services) Act. 2016 https://uidai.gov.in/images/the aadhaar act 2016.pdf (Accessed 15 Jan 2018). 
19. Lal SS, Sahu S, Wares F, et al. Intensified scale-up of public-private mix: a systems approach to tuberculosis care and control in India. Int $J$ Tuberc Lung Dis 2011;15:97-104.

20. Nagaraja SB, Achanta S, Kumar AM, et al. Extending tuberculosis notification to the private sector in India: programmatic challenges? Int $J$ Tuberc Lung Dis 2014;18:1353-6.

21. Uplekar M, Juvekar S, Morankar S, et al. Tuberculosis patients and practitioners in private clinics in India. Int $J$ Tuberc Lung Dis 1998;2:324-9.

22. Satpati M, Burugina Nagaraja S, Shewade HD, et al. TB notification from private health sector in delhi, india: challenges encountered by programme personnel and private health care providers. Tuberc Res Treat 2017:2017:1-9.

23. Govt launches mobile app to make TB notification simpler. http:// www.dnaindia.com/india/report-govt-launches-mobile-app-to-maketb-notification-simpler-1970345 (Accessed 28 Apr 2018).

24. Amdekar $Y$. Consensus statement on childhood tuberculosis working group on tuberculosis, Indian Academy of Pediatrics (IAP) Indian Pediatr. 2010;41 https://www.indianpediatrics.net/jan2010/41.pdf.

25. Ramachandran G, Kumar AK, Bhavani PK, et al. Pharmacokinetics of first-line antituberculosis drugs in hiv-infected children with tuberculosis treated with intermittent regimens in India. Antimicrob Agents Chemother 2015;59:1162-7.

26. Medical Council of India ND. INDIAN MEDICAL COUNCIL

(Professional Conduct, Etiquette and Ethics) Regulations,. 2002 https://www.mciindia.org/documents/rulesAndRegulations/Ethics Regulations-2002.pdf (Accessed 28 Apr 2018).

27. Gazette. Ministry of health and family welfare M 2018. notification. 2018 http://egazette.nic.in/WriteReadData/2018/183924.pdf (Accessed 28 Apr 2018)

28. Central TB Division, Directorate General of Health services, Ministry of Health and Family Welfare, Nirman Bhavan ND. National strategic plan for tuberculosis elimination 2017-2025. 2017:110-108 (Accessed 26 Apr 2018).

29. An Innovative Intervention in Indian Private Sector. Initiative for promoting affordable quality TB Tests (IPAQT). http://iammdelhi.com/ wp-content/uploads/2017/09/IPQAT-Dr-manjot-kaur.pdf (Accessed 8 May 2018).

30. Pai M. Promoting affordable and quality tuberculosis testing in India. J Lab Physicians 2013;5:1-4. 